\title{
Estimation of the impact duration for several types of structures
}

\author{
E. Jacquelin ${ }^{\mathrm{a}, *}$, S. Pashah ${ }^{\mathrm{b}}$, J.P. Lainéc ${ }^{\mathrm{and}}$ M. Massenzio ${ }^{\mathrm{a}}$ \\ ${ }^{a}$ Université de Lyon, F-69622, Lyon,France - LBMC, UMR-T9406, Université Lyon 1, Villeurbanne, France- \\ IFSTTAR, Bron, France \\ ${ }^{\mathrm{b}}$ Department of Mechanical Engineering, King Fahd University of Petroleum and Minerals, Box 1095 KFUPM, \\ Dharan 31261, Saudi Arabia \\ ${ }^{\mathrm{c}}$ Université de Lyon, F-69622, Lyon,France - Ecole Centrale de Lyon, CNRS, LTDS, UMR 5513, F-69134, Ecully, \\ France
}

Received 1 February 2011

Revised 16 September 2011

\begin{abstract}
The impact duration between two structures is not easy to forecast. Indeed it depends on many parameters including local parameters as well as structural parameters which characterize the dynamics of the structures.

In this paper we will show that it is possible to have a prior estimation of the impact duration. In fact the simulations show that this parameter is connected to some characteristics of a specific model, the so-called antioscillator model, even for the complex case of the impact between two flexible structures. All the simulations carried out on different cases show the close relationship between the first impact duration and the antioscillator characteristics. However some limitations exist and have been highlighted.
\end{abstract}

\section{Introduction}

The design of structures under low velocity impact requires to take the local problem of contact into account and the global description of the structures as well [1]: the cost of numerical calculations can be very high. A first step to decrease the number of degrees of freedom (dof) consists in modelling the interaction through a contact law (such as Hertz law) [2]. However for a complex structure described by a finite element model, the number of dof may be still large. So a second step has been proposed to represent the structure through a simplified model such as a single dof model $[3,4]$. However the application of such models is limited because they are not able to account for some dynamic aspects of the response.

Therefore Jacquelin et al. [5-7] proposed to extend the simplified models by describing a structure with its socalled "antioscillators" (AO): the AO model is an alternative to the traditional modal model. It has been shown that the AO model parameters may be derived easily from a finite element model of the structure [7].

The model used for simulating an impact event should be optimized according to the type of the response: a quasi-static response requires very few degrees of freedom (dof) contrary to a dynamic response. So to select the relevant model, the nature of the impact should be predicted. An interesting indicator is the comparison between the impact duration and the period of the first eigenmode: the main issue is to estimate the impact duration before any calculation. On the other hand, Christoforou et al. [8] proposed three non-dimensional parameters which control the impact event, and Olsson [9] studied the mass effect on the impact.

The objective of this paper is to propose a prior estimation of the impact duration based on the target/projectile characteristics: a relation to the AO characteristics will be particularly studied.

\footnotetext{
${ }^{*}$ Corresponding author. E-mail: eric.jacquelin@univ-lyon1.fr.
} 


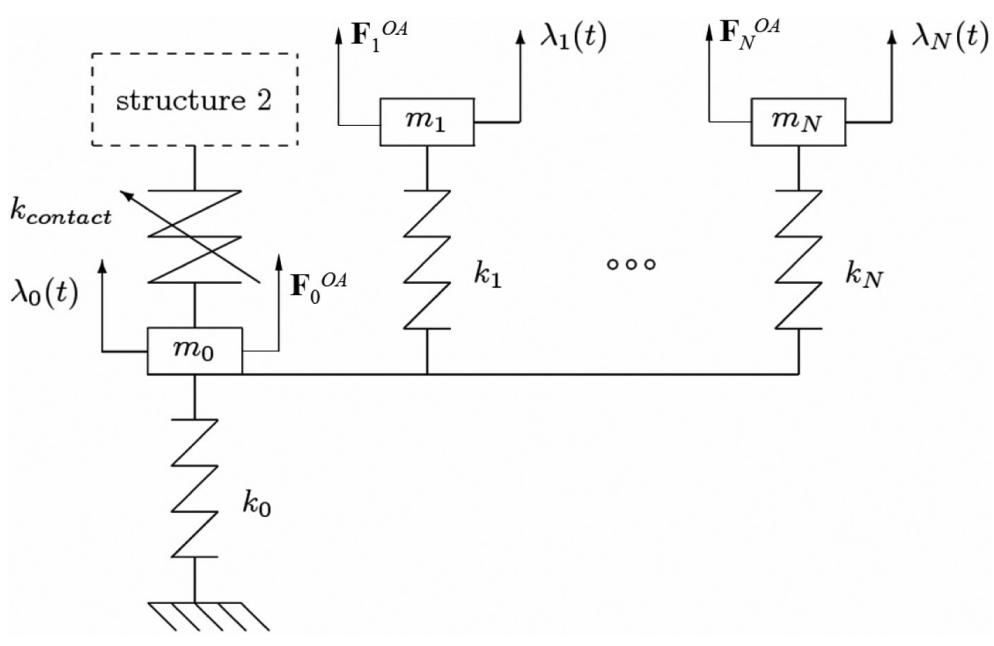

Fig. 1. Model of a structure impacted by another structure.

In this paper a brief presentation of the main features of the AO model will provided first. Then the impact duration estimation is done on different systems "target-projectile": firstly this paper deals with simple systems (e.g. rigid structure against flexible structure) and some results related to these simple systems have been presented in [10] but a deeper analysis is included in this paper. Finally a study on more complex systems (impact between two flexible structures) is done to estimate the impact duration.

\section{Antioscillator model (AO model)}

The objective of this section is not to describe precisely the antioscillator model: this has been previously done in the companion papers [5-7]. The goal of this section is to highlight the main features of the method.

First, it has been established in [5] that every structure with an elastic behaviour may be reduced to the lumped masses depicted in Fig. 1 defined by a set of spring-mass systems $\left\{m_{i}, k_{i}\right\}_{i=0 . . N}$.

The AO model is associated to a chosen dof $\lambda_{0}$ : in this study $\lambda_{0}$ is the displacement at the impact point $P_{A R}$ along the direction of the impact. This model is easily derived from a first finite element (FE) model provided $\lambda_{0}$ is already a dof of the FE model. Then the relation between both model is:

$$
[X]=\sum_{i=0}^{N} \lambda_{i}(t) c_{i} \phi_{i}(x)
$$

where:

$\mathrm{X}$ is the FE dof vector, $N$ is the FE dof number.

- $\forall i>0, \phi_{i}$ are the eigenshapes associated with the beam subjected to an additional boundary condition at the impact location: $\lambda_{0}$ is constrained to be equal to. $\phi_{i}$ is referred to as a constraint shape and the circular eigenfrequencies are denoted $\omega_{i}$.

- $\phi_{\text {st }}$ is the solution to the static problem defined by a static force applied at the impact location $(x=L)$ such as the displacement at the impact location is equal to unity. However this solution may have no solution when the system has one or more rigid body modes: in that case $\phi_{\text {st }}$ is one of the rigid body modes such as $\lambda_{0}=1$.

- $\phi_{0}$ is the so-called residual mode such as:

$$
\phi_{0}(x)=\phi_{\mathrm{st}}(x)-\sum_{i=1}^{\infty} c_{i} \phi_{i}(x)
$$

with the coefficients $c_{i>0}$ are defined so that $\phi_{0}$ is orthogonal to each constraint shape with respect to the mass operator, and $c_{0}=1$. 
The following properties have been demonstrated [5-7]:

- When $N$ tends to infinity the AO model response converges to the actual structural response,

- The mass matrix is (obviously) directly diagonal,

- The natural frequency of each 1-dof system $\left\{m_{i}, k_{i}\right\}_{i=1 \ldots N}$ is an antiresonant frequency of the structure: this is why they are called antioscillators; accordingly, the model depends on the point $P_{A R}$ where the response is evaluated and on the choice of the measured dof $\lambda_{0}$ which corresponds to the mass $m_{0}$ displacement(see Fig. 1),

- The antioscillators are sorted according to their increasing frequencies;

- When there is no rigid body mode, the stiffness $k_{0}$ is the "static" stiffness, ie the force applied in the direction of dof $\lambda_{0}$ that produces a unit displacement for $\lambda_{0}$ : the structural displacement field for this load case is the static mode $\phi_{s t}$; otherwise $k_{0}$ is equal to zero,

- Let $\mathrm{M}(.,$.$) be the mass operator: the kinetic energy \mathrm{K}$ is then $\mathrm{K}=1 / 2 \mathrm{M}(v(t), v(t))$ where $v(t)$ is the velocity field at instant $t$; the mass $m_{s t}$ is defined as $m_{s t}=\mathrm{M}\left(\phi_{s t}, \phi_{s t}\right) ; m_{0}$ is defined as follows:

$$
m_{0}=m_{s t}-\sum_{i=1}^{N} m_{i}
$$

So, $m_{0}$ is called the "residual mass",

- When the structure is loaded only at point $P_{A R}$ along the direction of dof $\lambda_{0}$, the elements $\left\{F_{i}^{A O}\right\}_{i=1 . . N}$ are equal to zero,

- In [6] it has been shown that the parameters $\left\{m_{i}, k_{i}\right\}_{i=0 . . N}$ have a physical meaning and they may be identified from the apparent-mass frequency response function,

- In [7] it has been shown that the parameters $\left\{m_{i}, k_{i}\right\}_{i=0 . . N}$ may be easily derived from a previous finite element model of the structure: in practice, for low velocity impact simulations, very accurate results may be obtained with much less dof if an AO model is used instead of an FE model [11].

\section{Impact duration}

Time duration $T_{c}$ is an interesting characteristic to investigate the nature of the impact. Indeed, it is well-known that when $T_{c}$ is lower than the half of the first eigen-period, then the impact will be quasi-static and dynamic otherwise. Thus it is interesting to have a prior knowledge of $T_{c}$. Moreover, when $T_{c}$ is known, the load spectrum may be estimated and then, the most likely excited modes of the structure may be predicted: so the number of modes, or the number of $\mathrm{AO}$ required to estimate accurately the response may be derived easily.

During an impact event, two structures are involved, and the impact does not have the same consequence on both of them: $T_{c}$ may be lower than the first eigenfrequency of one structure and greater for the other structure.

The goal of the rest of this paper is to predict $T_{C}$. Different cases will be studied, and for each case a relationship between $T_{C}$ and the natural period of the first $\mathrm{AO}, T_{A O_{1}}$ will be addressed. Indeed, if one considers an axial impact between two identical free bars, it is well known that the impact duration is equal to the time required for the reflection of the extension stress waves along the bar:

$$
T_{c}=2 \times \frac{L}{c_{0}}
$$

where $L$ is the bar length and $c_{0}$ the wave speed. It is easy to calculate the natural eigen-frequency of the first $\mathrm{AO}[10]$ of a free bar. One obtains then the striking results:

$$
T_{c}=\frac{1}{2} T_{A O_{1}}
$$

The question is then to determine whether relation (5) is only a chance or may be extended to other cases of impact to forecast the impact duration. 
Table 1

beam and Hertz spring characteristics

\begin{tabular}{cccccc}
\hline$E(\mathrm{GPa})$ & $\rho$ (kg.m $\left.\mathrm{m}^{-3}\right)$ & $a(\mathrm{~mm})$ & $L(\mathrm{~m})$ & $k_{H}\left(\mathrm{~N} \cdot \mathrm{m}^{-1.5}\right)$ & $v_{0}\left(\mathrm{~m} \cdot \mathrm{s}^{-1}\right)$ \\
\hline 66.0 & 2500.0 & 14.1 & 1.0 & $1.510^{10}$ & $1.0-100.0$ \\
\hline
\end{tabular}

\subsection{Interaction law}

The impact duration is not a structure dependent parameter only: the interaction law may govern $T_{c}$ as well. In the following the interaction is modelled by a Hertz law, ie by a non-linear spring characterized by its stiffness $k_{H}$. As mentioned by Abrate [2] this approach is commonly used to predict the impact force and deformation history; he has suggested that a statically identified law is sufficient for a low velocity impact.

\subsection{Half-space case}

As defined by Hunter [12], an impact half-space case is the situation where the projectile mass is very small with respect to the mass of the impacted structure. Consequently there is little motion of the structure and then the impact response is considered to be localized $[1,8,13,14]$. Then the impacted structure may be modelled as a nonlinear spring characterizing the local deformation which is clamped at one end.

In this section a structure is supposed to hit an half space through a Hertz spring characterized by its stiffness $k_{H}$. Two projectiles are studied: a rigid projectile and a pinned-free beam.

When the projectile is rigid and characterized by its mass $m_{p}$, the impact duration may be easily estimated by linearizing (energy balanced model) the non-linear contact spring. The equivalent linearized stiffness is given by [2]:

$$
k_{\text {lin }}=\left(\frac{\pi}{3.21}\right)^{2} \times\left(m_{p} k_{H}^{4} v_{0}^{2}\right)^{1 / 5}
$$

where $v_{0}$ is the impact velocity.

Indeed, it is well-known that in that case, impact duration $T_{H S}$ is well estimated by the natural half-period of the oscillator $\left(m_{p}, k_{l i n}\right)[1]$, and is given by Eq. (7):

$$
T_{H S}=\pi \sqrt{\frac{m_{p}}{k_{l i n}}}=3.21\left(\frac{m_{p}^{2}}{k_{H}^{2} v_{0}}\right)^{1 / 5}
$$

This case of impact is the simplest one. Obviously relation (5) is not applicable because this structure has no antioscillator. This example shows the first limitation of relation (5): at least, one of the structure involved in the impact event must not be modelled as a rigid structure.

Consider now the impact of a pinned-free beam on a hemisphere (see Table 1); the beam has a square cross-section. The Hertz stiffness may be derived from the contact geometry and the mechanical properties of the beam and the hemisphere. Moreover, if the beam is impacted at its end, by comparing the kinetic energy of the pinned-free beam and the kinetic energy of an equivalent mass, this mass $m_{e q}$ is equal to one-third of the beam mass. Then at first sight, one would predict the time duration in the same manner:

$$
T_{c}^{\text {rigid }}=3.21\left(\frac{m_{e q}^{2}}{k_{H}^{2} v_{0}}\right)^{1 / 5}=0.1 \mathrm{~ms}
$$

However the simulation gives a result that is more than hundred times greater than the estimated duration $\left(T_{c}=13.2\right.$ $\mathrm{ms})$. Hence, the rigid mass approach is not valid.

To account for the dynamics of the beam, an AO model is considered (see Table 2 for the characteristics of the first five $\mathrm{AO})$.

In that case, $1 / 2 T_{A O_{1}}$ is equal to $15.2 \mathrm{~ms}$ that is a quite close estimation of the impact duration. Other simulations have been performed for a wide range of velocities and the same impact duration has been obtained: the impact duration seems not to be sensitive to the impact velocity. Such results have already been shown in [10] for another beam. All these results indicate that relation (5) is applicable in this case. 
Table 2

Characteristics of the pinned-free beam AO model

\begin{tabular}{cccccc}
\hline AO number & 1 & 2 & 3 & 4 & 5 \\
\hline mass $m_{i}(\mathrm{~g})$ & 101.3 & 25.3 & 11.3 & 6.3 & 4.0 \\
frequency $f_{A R}(\mathrm{~Hz})$ & 32.9 & 131.6 & 295.6 & 524.4 & 816.9 \\
\hline
\end{tabular}

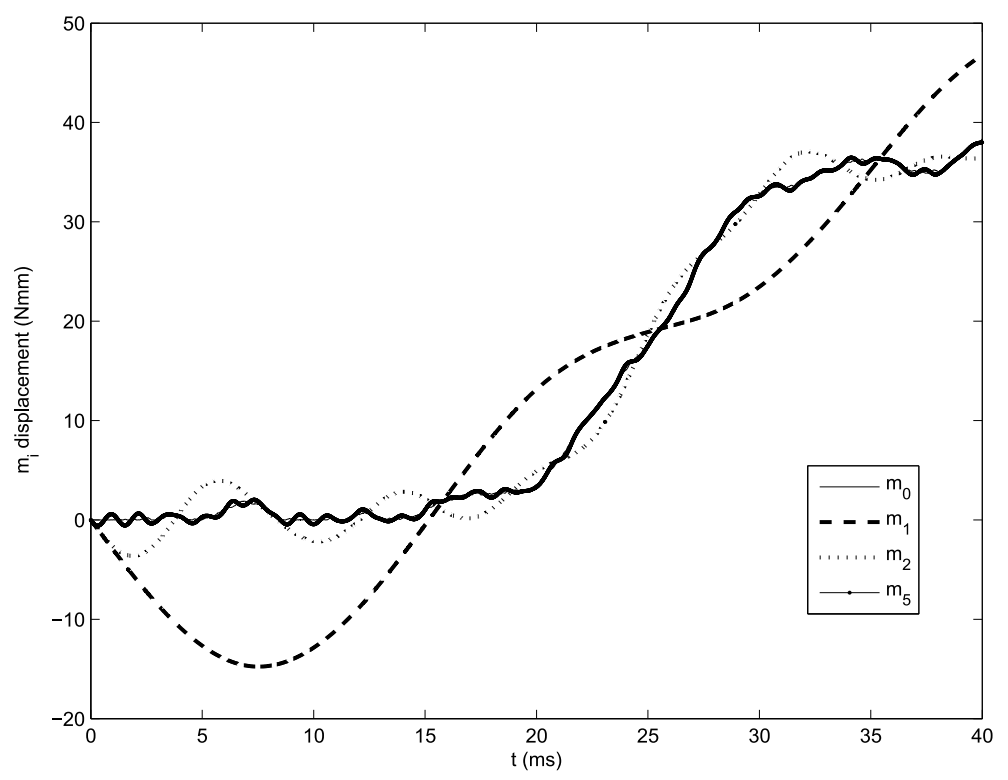

Fig. 2. $m_{0}, m_{1}, m_{2}, m_{5}$ displacement.

Figure 2 shows the movement of the AO masses. Note that from rank $i$ equal to 5 , the $m_{i}$ mass movement is very close to the movement of $m_{0}$, as shown in Fig. 2. That means that a truncation may be done up to this rank: the $\mathrm{AO}$ beyond this rank may have their mass gathered with the mass $m_{0}$. Then the AO model can provide not only a good estimation of the impact duration but also a simple model with very few of degree of freedom to simulate this impact event.

\subsection{Rigid structure against soft structure}

The next step in increasing the complexity level is to study the impact of a rigid structure on a flexible structure. A pinned-pinned beam impacted by a rigid sphere will illustrate this study. The beam properties are listed in Table 1 .

It was mentioned above that the impact duration is compared to the half-period of the first AO. However, Fig. 1 shows that one problem to be solved is to identify the first AO. Indeed, during the impact, the set \{projectile, Hertz spring \} may be viewed as an additional antioscillator (see Fig. 1 where structure 2 is the rigid sphere) provided to linearize the contact spring as indicated in the previous subsection (see Eq. (6)): an additional AO frequency, $f_{A A O}$, may be defined by

$$
f_{A A O}=\sqrt{\frac{m_{p}}{k_{l i n}}}
$$

Depending on the projectile mass and the Hertz stiffness, this additional AO can be sorted by comparing the additional $\mathrm{AO}$ frequency to $f_{A O 1}$. So in the following it will be studied how the time duration varies when $f_{A A O}$ varies whereas $f_{A O 1}$ remains constant.

There are three ways to make $f_{A A O}$ vary: by varying either $m_{p}$ or $k_{H}$ or $v_{0}$. The latter parameter can not be increased too much for staying in the low velocity impact range; moreover $v_{0}$ is set to the power $1 / 5$ (see Eq. (6)): therefore the variation will be slow. For example $f_{A A O}$ is multiplied by 4 when $v_{0}$ is increased 1000 times (ie 


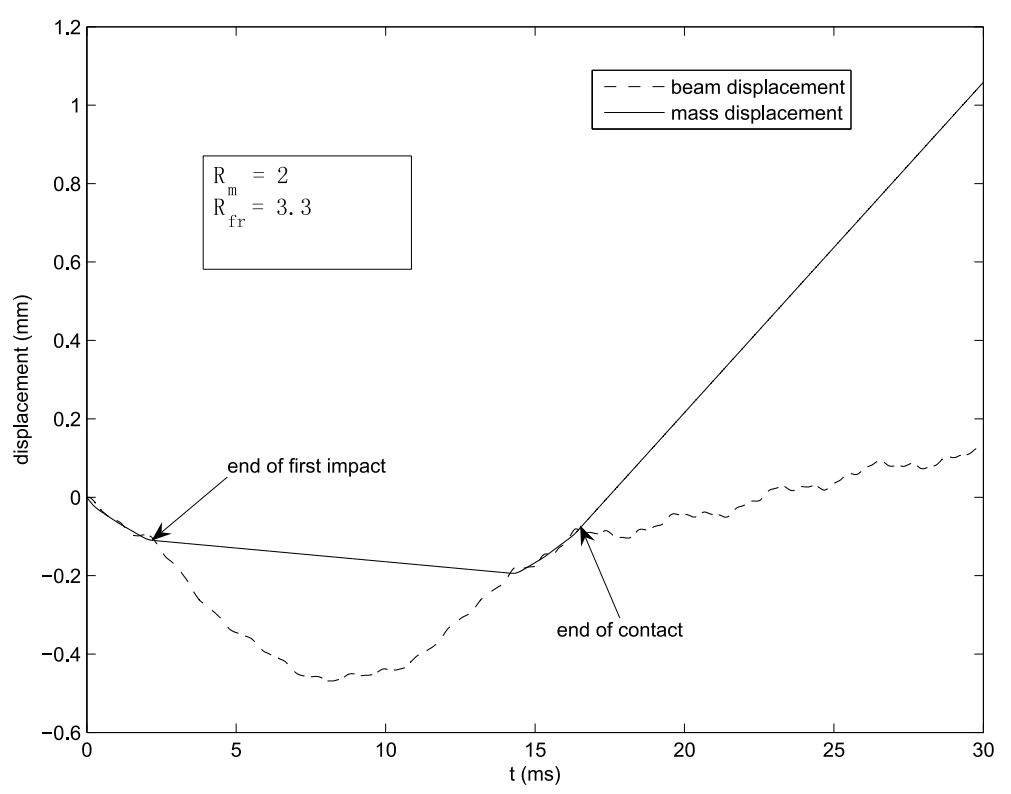

Fig. 3. Mass-beam impact: beam mid-span displacement and mass displacement.

from $0.1 \mathrm{~m} . \mathrm{s}^{-1}$ to $100 \mathrm{~m} \cdot \mathrm{s}^{-1}$ ). In the following the impact velocity is set equal to $1 \mathrm{~m} . \mathrm{s}^{-1}$. Therefore, only the influence of $m_{p}$ and $k_{H}$ will be studied. So a sensitivity analysis has been done in function of two non-dimensional parameters:

- frequency ratio $R_{f r}$ :

$$
R_{f r}=\frac{f_{A A O}}{f_{A O 1}}
$$

When this parameter is less than unity the projectile associated with the linearized Hertz spring can be considered as the first antioscillator.

- mass ratio $R_{m}$ :

$$
R_{m}=\frac{m_{p}}{m_{A O 1}}
$$

When this parameter is far below unity one can consider that the projectile mass is negligible compared to the beam mass. This parameter characterizes the influence of the masses involved in the impact.

Figure 3 shows that an impact duration definition issue arises: sometimes multiple contacts occur, and one has to distinguish the first impact duration $\left(T_{c 1}\right)$ and the total impact duration $\left(T_{c}\right.$ tot $)$ which starts at the beginning of the first impact and ends at the end of the last impact. Thus, considering Fig. 3, $T_{c 1}$ is equal to $2.3 \mathrm{~ms}$ whereas $T_{c}$ tot is equal to $16.5 \mathrm{~ms}$. In the following the impact duration will referred to the first impact duration when multiple impact occur: $T_{c}=T_{c 1}$.

Some simulations have been performed for several $\left(R_{m}, R_{f r}\right)$. For each simulation, the first impact duration and the total impact duration are evaluated and represented in Fig. 4: for each mass ratio, these durations are given as a function of the frequency ratio. To study their trends, $T_{c}$ and $T_{c}$ proj are depicted as well as $T_{Q S}$ which is defined as follows:

$$
T_{Q S}=\pi \sqrt{\frac{m_{p}}{k_{e q}}}
$$

with:

$$
k_{e q}=\frac{k_{0} k_{l i n}}{k_{0}+k_{l i n}}
$$



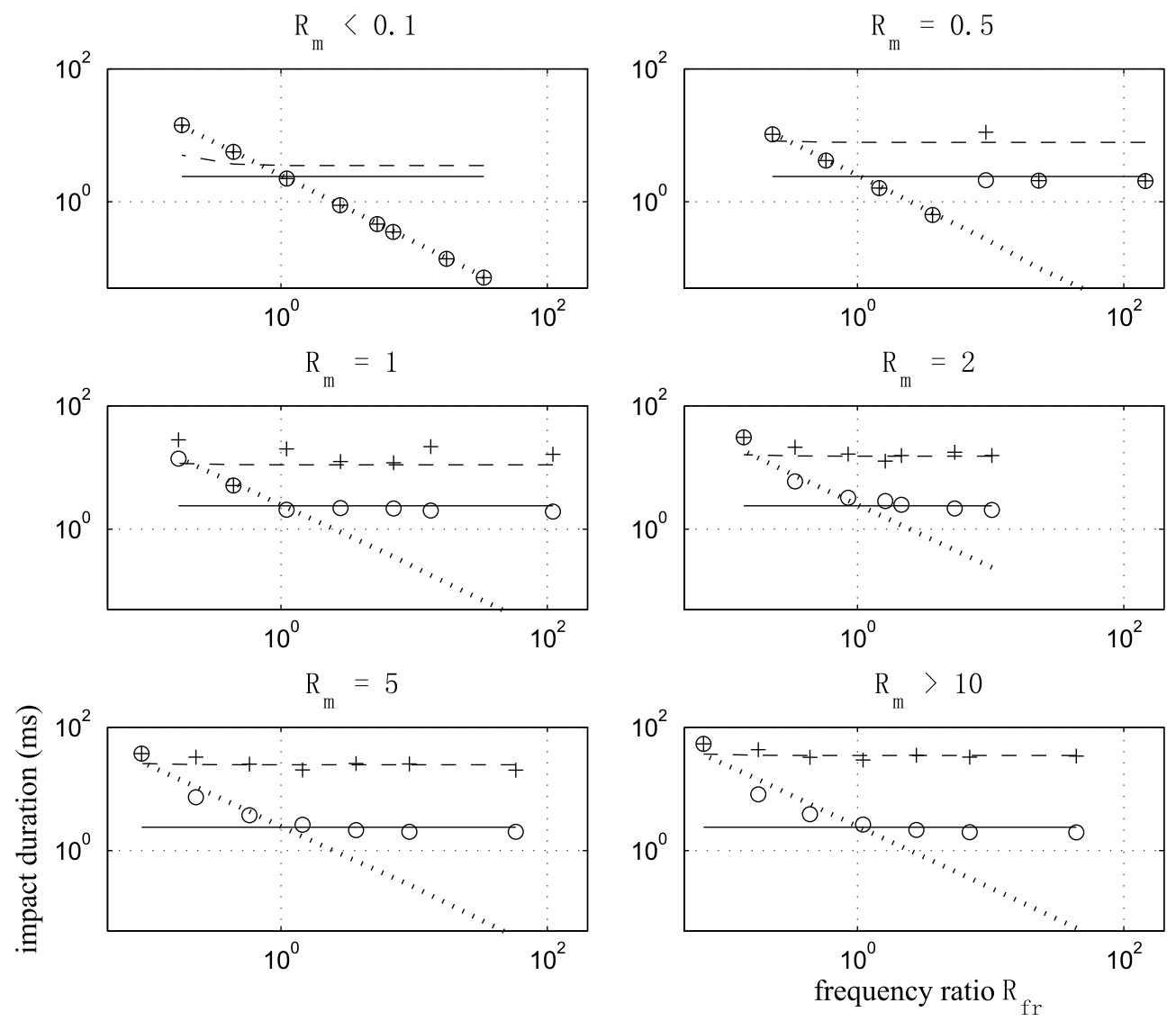

Fig. 4. Impact duration sensibility: "O": first impact duration; "+": total impact duration; solid line: $1 / 2 T_{A O 1}$; doted line: $1 / 2 T_{A A O}$; dashed line: $T_{Q S}$.

$T_{Q S}$ corresponds to a quasi-static response of the beam which is modelled as a massless spring characterized by its static stiffness $k_{0}$.

The results depend on the mass ratio. When the mass ratio is less than 0.1 , it appears that the impact duration corresponds to $1 / 2 T_{A A O}$ even for a frequency ratio greater than unity: i.e. the impact duration is not always controlled by the first AO in that case. This may be easily justified. Indeed in that case the projectile mass may be considered negligible compared to the static mass of the beam. Then the beam has no noticeable response and may be considered as an half-space compared to the projectile: we have seen in the previous subsection that in that case, relation (5) is not applicable and the impact duration is well estimated by relation (7).

When the mass ratio is between 0.1 and 10 , the impact duration switches between $1 / 2 T_{A A O}$ and $1 / 2 T_{A O 1}$ : the switch occurs around a frequency ratio equal to unity. That means that the impact duration may be estimated by the half period of the first antioscillator which is either the additional one when $R_{f r}$ is lower than one, or the structural one when $R_{f r}$ is greater than one. Then relation 5 is valid in this mass ratio range.

When the mass ratio is greater than 10 the impact duration is clearly well-estimated by $T_{Q S}$ : in that case relation (5) is not applicable. This may be explained easily because when $R_{m}$ is greater than 10 , the mass of the beam may be neglected compared with the projectile mass: the beam can be modelled by a massless beam.

All the previous results are summarized in Fig. 5 and one can see that relation (5) is applicable when the mass ratio is in the range $[0.1 ; 10]$. When the mass ratio is lower than 0.1 , the impact duration is well estimated by $T_{H S}$, while it is well estimated by $T_{Q S}$ when the mass ratio is greater than 10 , as explained above.

As to the total impact duration, the key parameter is the mass ratio whereas the frequency ratio has weak influence. For mass ratio greater than unity, generally multiple impacts occur, thus $T_{c 1}$ and $T_{c}$ tot are not equal. In that case, the total impact duration is well estimated by $T_{Q S}$. This means that the projectile mass and the beam stiffness globally 


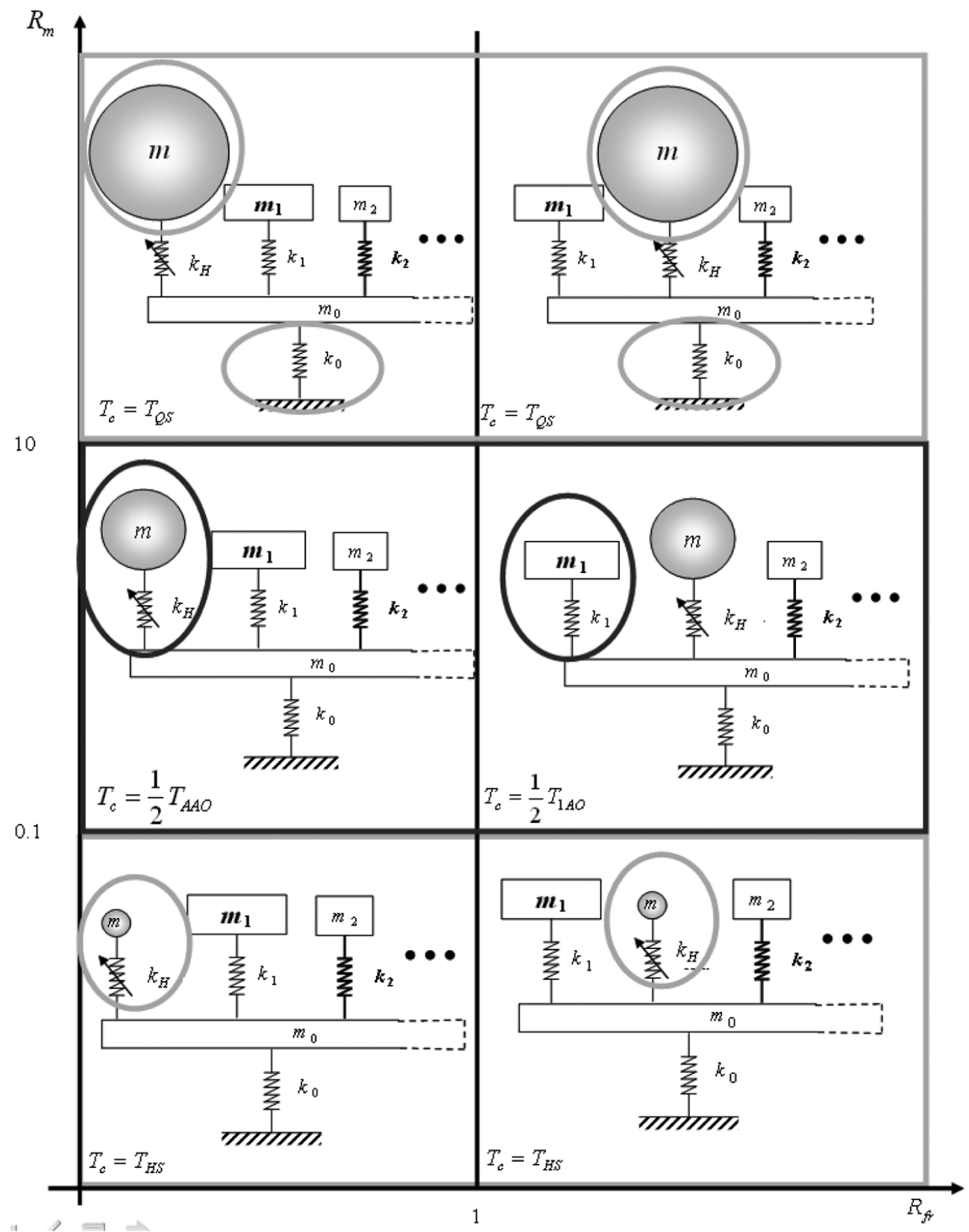

Fig. 5. PP beam/mass Impact duration: summary.

control the movement during the impact event. When the mass ratio is lower than 0.1 , only one impact occurs and then, as explained above, the projectile still controls the impact as the beam behaves as an half-space. When the mass ratio is between 0.1 and 1 , one has an intermediate case, and it is difficult to draw some conclusions because it is not obvious to predict whether the impact will be multiple or not.

\subsection{Impact between Flexible structures}

The pinned-pinned beam (PP beam: structure 1) studied in the previous subsection is now impacted by a pinnedfree beam (PF beam: structure 2). The properties of both beams are similar except the length and the density which 

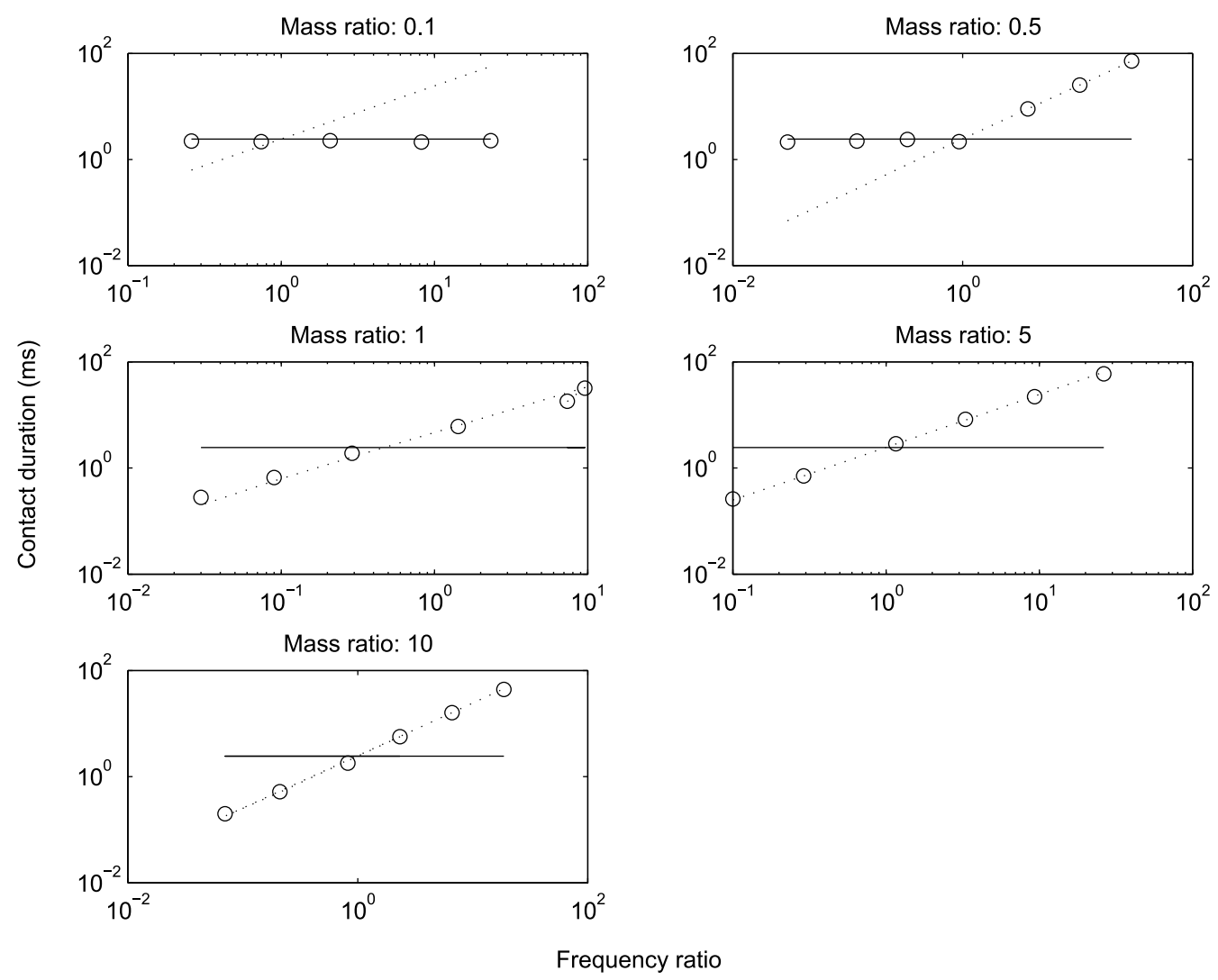

Fig. 6. PF beam against PP beam - Impact duration sensibility: “O”: first impact duration; solid line: $1 / 2 T_{A O P P} ;$ doted line: $1 / 2 T_{A O} P F$.

are adjustable parameters to make the AO parameters vary. Each beam is impacted at its mid-span.

Moreover the contact stiffness is supposed to be high enough to consider that the residual masses of both structures are stuck during the contact phases, ie they have almost the same displacement.

Our aim is to correlate the first contact duration to the AO first half-period of one structure. Therefore, a parametric study is done by varying two non-dimensional parameters:

- frequency ratio $R_{f r 1 / 2}$ :

$$
R_{f r 1 / 2}=\frac{f_{A O, s t r 1}}{f_{A O, s t r 2}}
$$

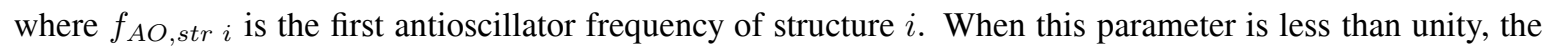
first antioscillator is given by structure 1: then this parameter indicates which structure has the lowest first antioscillator.

- mass ratio $R_{m 1 / 2}$ :

$$
R_{m 1 / 2}=\frac{m_{A O 1}}{m_{A O 2}}
$$

This parameter aims to study the influence of the mass of one structure compared to the other structure.

The results mentioned in the previous subsection show that when the mass ratio is not in the range $[0.1 ; 10]$ relation (5) has no chance to be satisfied. So in the following $R_{m 1 / 2}$ will remain in this range. Note that to perform simulations on a wide range of structures ie for a wide range of frequency ratio, the characteristics of structure 1 (PP-beam) were unchanged whereas the characteristics of structure 2 (PF-beam) varied.

In Fig. 6, the time duration is given as a function of the frequency ratio, for a given mass ratio. The evolution of $f_{A O, s t r 1}$ and $f_{A O, s t r 2}$ has been also represented. One can notice that the contact duration is given by the first AO 

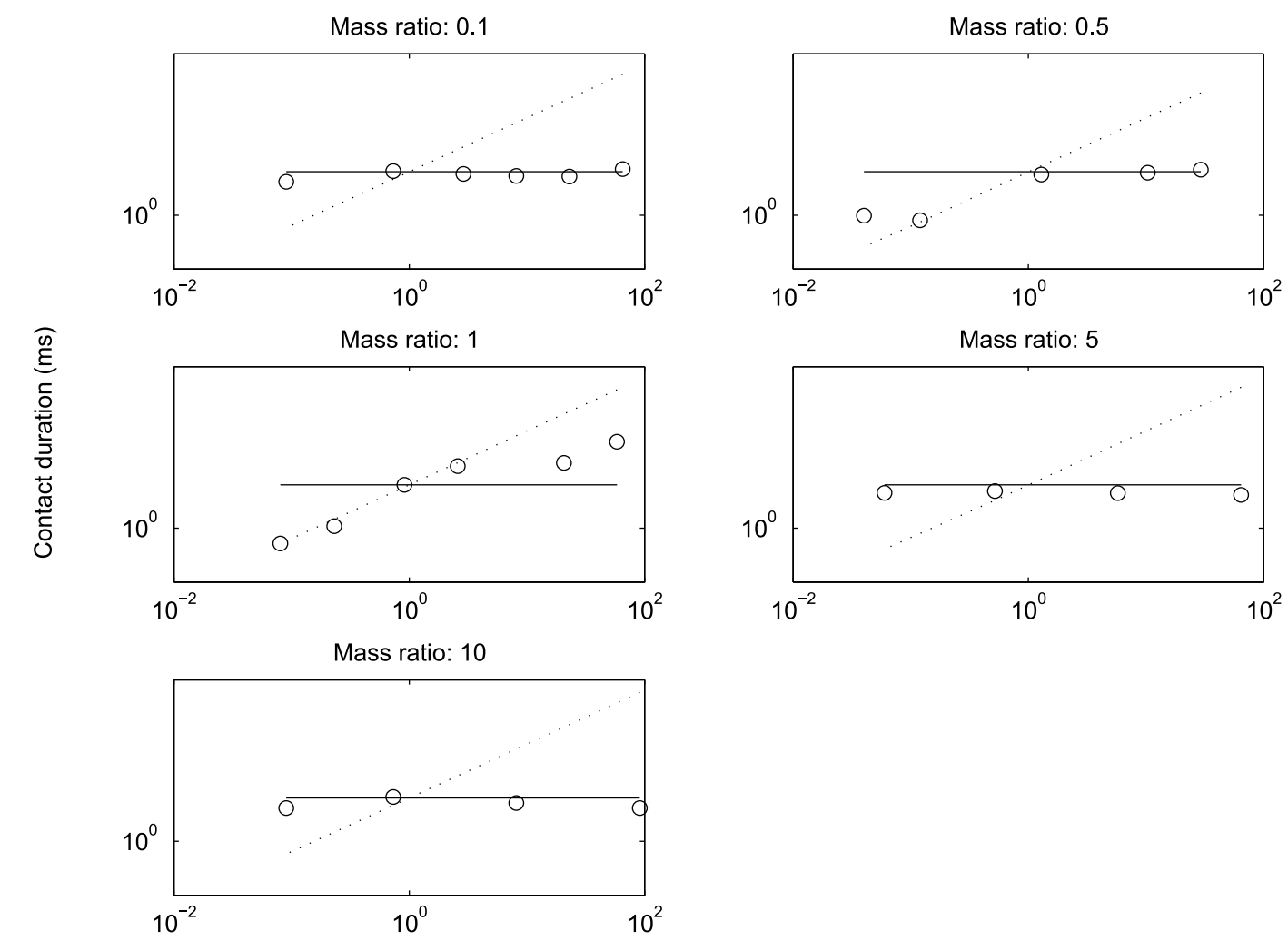

Frequency ratio

Fig. 7. PF beam against FF beam - Impact duration sensibility: "O": first impact duration; solid line: $1 / 2 T_{A O} P F$; doted line: $1 / 2 T_{A O} F F$.

half-period of one structure. We can see that the contact duration depends strongly on the mass ratio: when this ratio is lower than $1 / 2$ the $\mathrm{PP}$ beam first $\mathrm{AO}$ controls the contact duration whereas for a ratio greater than $1 / 2$ the $\mathrm{PF}$ beam first $\mathrm{AO}$ controls the first impact duration. Around a mass ratio equal to $1 / 2$, there is a transition between the previous two situations: this mass ratio is then a critical mass ratio. Consequently, Fig. 6 shows that the conclusions done previously as to the influence of the first AO frequency sorting is no longer available. However, this example shows that relation (5) is still applicable. However the problem is to determine which structure controls the impact duration: unfortunately the frequency ratio does not seem to be able to solve this issue.

It must be noticed that the results shown in Fig. 6 are obtained by varying the characteristics of the PF beam: the numerator of the mass ratio and the frequency ratio remains constant while the denominator varies. It has been verified that the results are similar when the PF beam characteristics remain constant while the PP beam characteristics vary.

Additional studies have been done. Some other boundary conditions have been considered:

- PF beam against FF beam,

- PF beam against PF beam

The results are shown in Figs 7-8.

Once more the equality between $T_{c}$ and $1 / 2 T_{A O, s t r}$ appears clearly in these two other cases. There is also a transition mass ratio (around 0.5-1).

Sometimes, the problem with an impact between two flexible structures comes from the difficulty to distinguish one impact from multiple impacts which are very close. However, when it appeared that independent impacts occurred, each contact duration is almost constant and is equal to $1 / 2 T_{A O, s t r}$, as shown in Fig. 9. 

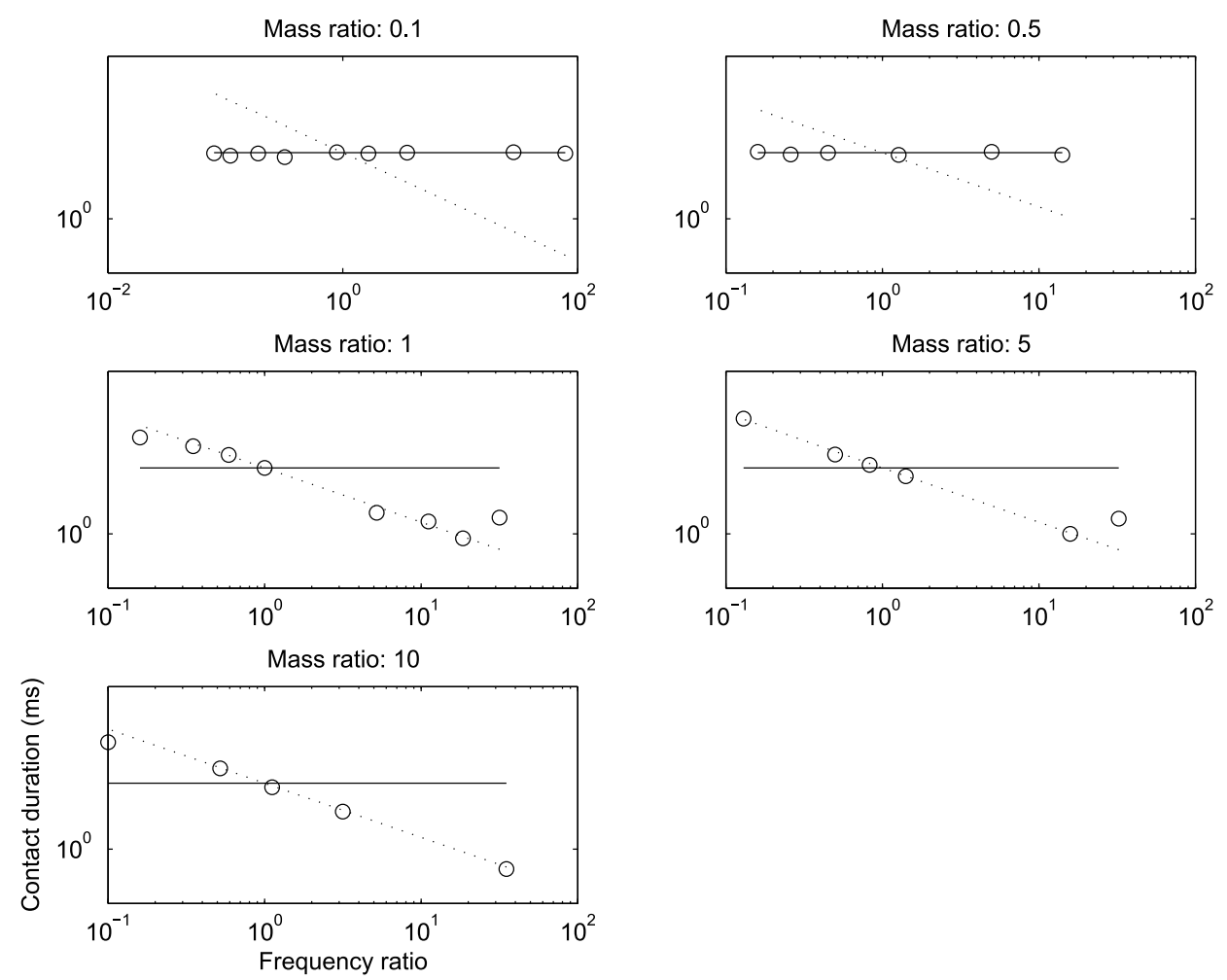

Fig. 8. PF beam against PF beam - Impact duration sensibility: “o": first impact duration; solid line: $1 / 2 T_{A O} P F 1$; doted line: $1 / 2 T_{A O P F}$.

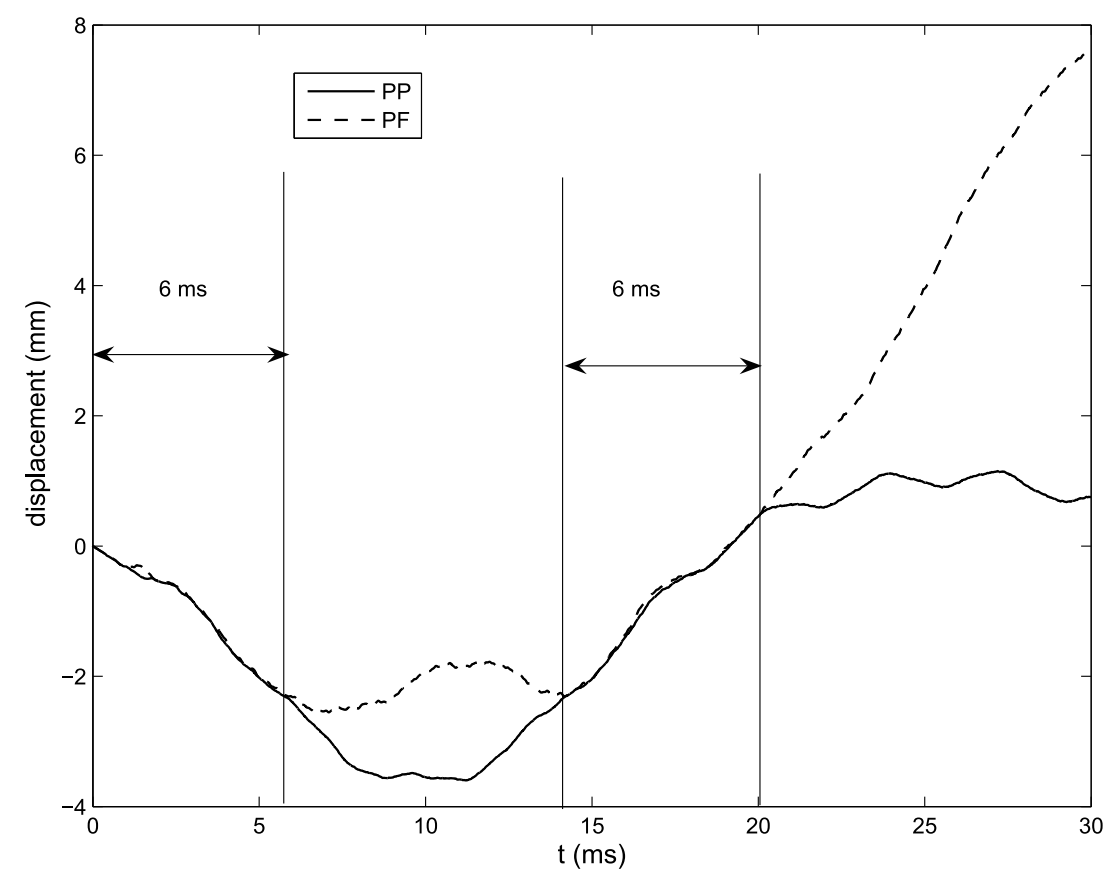

Fig. 9. PF beam against PP beam $-R_{m}=1-R_{f}=2.6-$ mid span displacement. 


\section{Conclusion}

This study was about estimating the contact duration. It has been highlighted that the total contact duration is different from the first impact duration when multiple impacts occur. This paper focussed on the first impact duration and its relation with the characteristics of the first AO of the structures involved in the impact event. This comes from a theoretical result concerning the axial impact between two identical bars: the first AO half-period of the bars is equal to the theoretical contact duration.

To expand this result to other structures we addressed several cases of impact:

- The impact between a flexible structure and a half-space medium confirms this results.

- The impact between a flexible and a rigid structure indicated that an additional AO must be considered: the rigid mass associated with the linearized contact spring. The first impact duration is then equal to either the structural first AO half-period or the additional first AO half-period only if the mass ratio is not too small or too large.

- The impact between two flexible structures is much more complex and has been studied for a mass ratio between 0.1 and 10. The results showed that the first impact duration seems to be the first $\mathrm{AO}$ half-period of one of the structures. However there is not a clear criterion which indicates which structure controls the impact duration.

The simulations on different kinds of tested structures show that the antioscillator characteristics are a good indicator to forecast the first impact duration. Further studies must be performed on more complex structures to find which structure controls the impact duration.

\section{References}

[1] W.J. Stronge, Impact mechanics, Cambridge University press, 2000.

[2] S. Abrate, Impact on Composite Structures, Cambridge University Press, 1998.

[3] R.B. Bucinell, R.J. Nuismer and J.L. Koury, Response of composite plates to quasi-static impact events. Composite Materials: Fatigue and Fracture ASTM STP 11103 (1991), 528-549.

[4] K.N. Shivakumar, W. Elber and W. Illg, Prediction of impact force and duration due to low-velocity impact on circular composites laminates, Journal of Applied Mechanics 52 (September 1985), 674-680.

[5] E. Jacquelin, J.P. Lainé, A. Bennani and M. Massenzio, A modelling of an impacted structure based on constraint modes, Journal of Sound and Vibration 301(3-5) (April 2007), 789-802.

[6] E. Jacquelin, J.P. Lainé, A. Bennani and M. Massenzio, The anti-oscillator model parameters linked to the apparent mass frequency response function, Journal of Sound and Vibration 312(4-5) (May 2008), 630-643.

[7] E. Jacquelin, J.P. Lainé and M. Massenzio, The antioscillator model and the finite element method, Journal of Sound and Vibration 324 (2009), 317-331

[8] A.P. Christoforou and A.S. Yigit, Effect of flexibility on low velocity impact response, Journal of Sound and Vibration 217(3) (1998), $563-578$.

[9] R. Olsson, Mass criterion for wave controlled impact response of composite plates, Composite, Part A 31(8) (2000), 879-887.

[10] S. Pashah, M. Massenzio and E. Jacquelin, Structural response of impacted structure described through anti-oscillators, International Journal of Impact Engineering 35 (2008), 471-486.

[11] E. Jacquelin, On the rigid projectile model on low velocity impact, International Journal of Impact Engineering 36 (2009), $1006-1011$.

[12] S.C. Hunter, Energy absorbed by elastic waves during impact, Journal of the Mechanics and Physics of Solids 5(3) (1957), 162-171.

[13] A.P. Christoforou and A.S. Yigit, Characterization of impact in composite plates, Composite Structure 43 (1998), 15-24.

[14] A.S. Yigit and A.P. Christoforou, Impact dynamics of composite beams, Composite Structures 32(1-4) (1995), 187-195. 

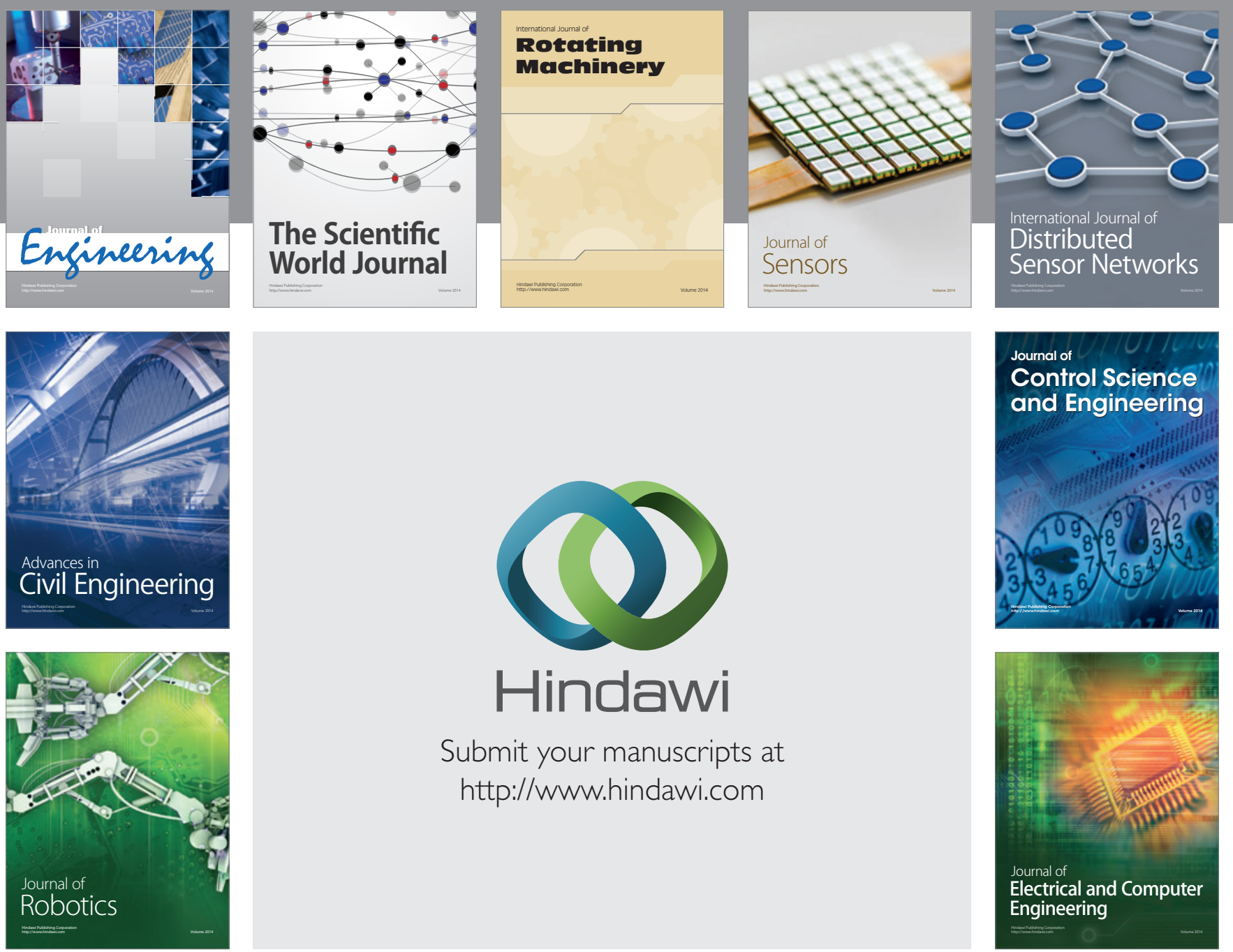

Submit your manuscripts at

http://www.hindawi.com
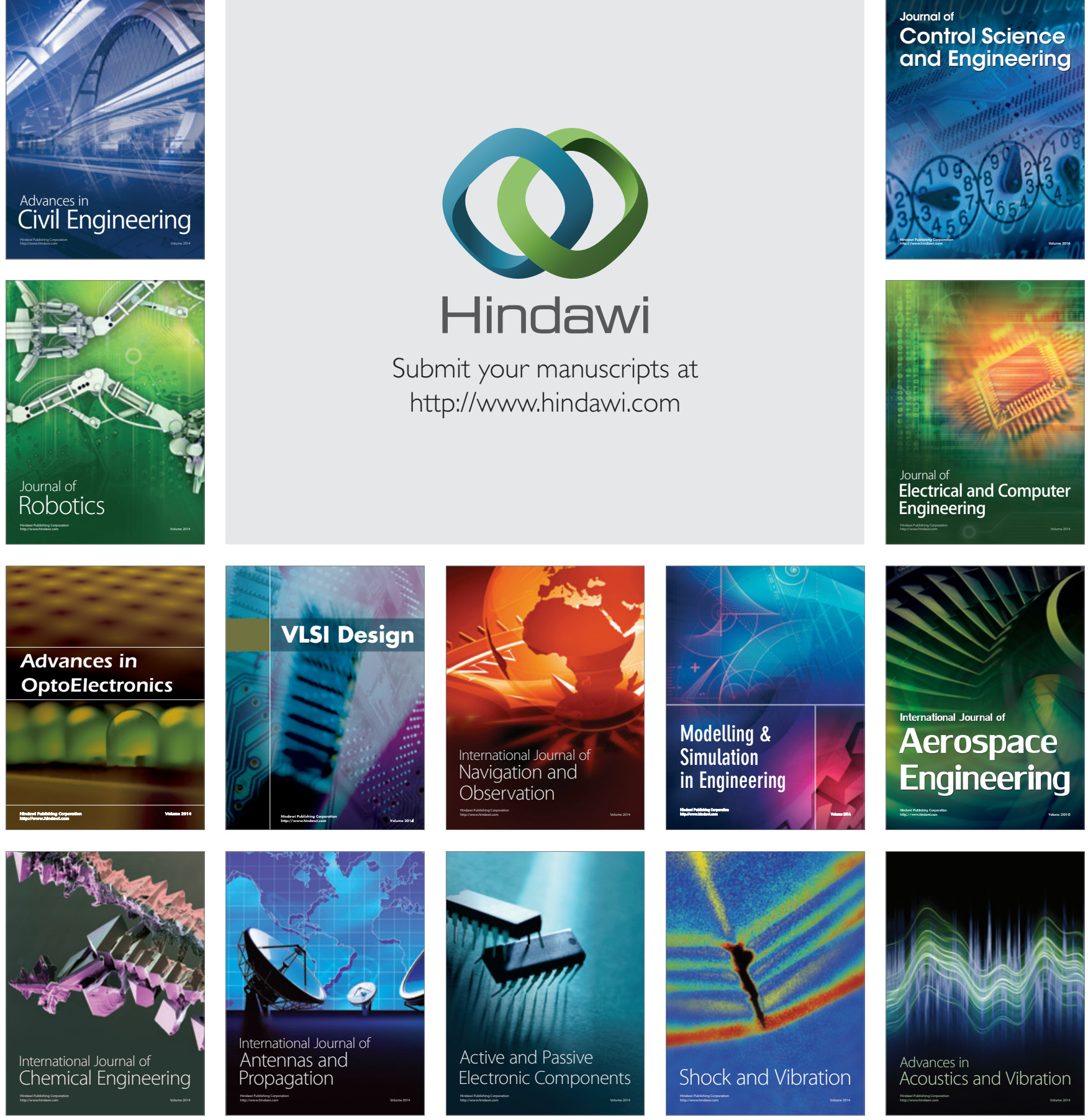